\title{
Correspondence Principle in Quantum Gravity
}

\author{
Kirill A. Kazakov a b* \\ ${ }^{a}$ Moscow State University, Physics Faculty, Department of Theoretical Physics, \\ 117234, Moscow, Russian Federation \\ bUppsala University, Physics Department, \\ SE-751 21, Uppsala, Sweden
}

The problem of consistent formulation of the correspondence principle in quantum gravity is considered. The usual approach based on the use of the two-particle scattering amplitudes is shown to be in disagreement with the classical result of General Relativity given by the Schwarzschild solution. It is shown also that this approach fails to describe whatever non-Newtonian interactions of macroscopic bodies. An alternative interpretation of the correspondence principle is given directly in terms of the effective action. Gauge independence of the $\hbar^{0}$ part of the one-loop radiative corrections to the gravitational form factors of the scalar particle is proved, justifying the interpretation proposed. Application to the black holes is discussed.

Establishing the correspondence between classical and quantum modes of description in the case of the theory of gravity displays features quite different from those encountered in other theories of fundamental interactions. Namely, the usual limiting procedure of the transition from quantum to classical theory turns out to be inapplicable in General Relativity. In electrodynamics, e.g., the quantum potential, defined as the Fourier transform (with respect to the momentum transfer from one particle to another) of a suitably normalized two-particle scattering amplitude, takes the form of the Coulomb law when the momentum transfer becomes small as compared with the particles' masses, so the above mentioned procedure is accomplished by tending the masses to infinity. Contrary to this, in General Relativity, the radiative corrections do not disappear in the limit: particle masses $\rightarrow \infty$, because strength of the gravitational interaction of particles is proportional to their masses. In fact, the relative value of the radiative corrections to the classical Newton law, corresponding to the logarithmical contribution to the gravitational form factors of the scalar particle, is independent of the scalar particle mass [ 1]. Yet, this contribution is proportional to the Planck

*E-mail: Kirill.Kazakov@fysik.uu.se constant $\hbar$. Newton's theory is therefore correctly reproduced in the formal limit $\hbar \rightarrow 0$.

There is, however, a still more important aspect of the correspondence between classical and quantum pictures of gravitation. The Einstein theory, being essentially nonlinear, demands quantum theory to reproduce not only the Newtonian form of the particle interaction, but also all the nonlinear corrections predicted by General Relativity. In this respect, the above-mentioned peculiarity of the gravitational interaction, namely, its proportionality to the masses of particles, is manifested in the fact (also pointed out in Ref. [ 1]) that, along with true quantum corrections (i.e., proportional to the Planck constant $\hbar$ ), the loop contributions also contain classical pieces (i.e., proportional to $\left.\hbar^{0}\right)$. Thus, an important question arises as to relationship between these classical loop contributions and the classical predictions of General Relativity.

In analogous situation in the Yang-Mills theories, the correct correspondence between classical and quantum theories is guaranteed by the fact that all radiative corrections to the particle form factors disappear in the limit: masses $\rightarrow \infty$, thus providing the complete reduction of a given quantum picture to the corresponding nonlinear classical solution. It is claimed in Ref. [ 
1] that when collected in the course of the construction of the gravitational potential from the one-particle-reducible Feynman graphs, the aforesaid classical contributions exactly reproduce the post-Newtonian terms given by the expansion of the Schwarzschild metric in powers of $r_{g} / r$, where $r_{g}$ is the gravitational radius.

However, as will be shown presently, the value of the potential found in Ref. [ 1] disagrees with the classical result given by the Schwarzschild solution. The latter has the form

$$
\begin{aligned}
& d s^{2}=\left(1-\frac{r_{g}}{r}\right) c^{2} d t^{2}-\frac{d r^{2}}{1-\frac{r_{g}}{r}} \\
& -r^{2}\left(d \theta^{2}+\sin ^{2} \theta d \varphi^{2}\right),
\end{aligned}
$$

where $\theta, \varphi$ are the standard spherical angles, $r$ is the radial coordinate, and $r_{g}=2 G M / c^{2}$ is the gravitational radius of a spherically-symmetric distribution of mass $M$. The form of $d s^{2}$ given by Eq. (ID) is fixed by the requirements $g_{t i}=0$, $i=r, \theta, \varphi, g_{\theta \theta}=-r^{2}$. To compare the two results, however, one has to transform Eq. (11) to the DeWitt gauge

$\eta^{\mu \nu} \partial_{\mu} g_{\nu \alpha}-\frac{1}{2} \eta^{\mu \nu} \partial_{\alpha} g_{\mu \nu}=0$,

used in Ref. [1].

The $t, \theta, \varphi$-components of Eq. (2) are already satisfied by solution (11). To meet the remaining condition, one substitutes $r=f(\tilde{r})$. A simple calculation shows that the function $f(\tilde{r})$ should expand as

$$
f(\tilde{r})=\tilde{r}\left[1+\frac{1}{2} \frac{r_{g}}{\tilde{r}}+\frac{1}{2}\left(\frac{r_{g}}{\tilde{r}}\right)^{2}+\cdots\right]
$$

at large $\tilde{r}$ for Eq. (2) to be satisfied to the order $r_{g}^{2} / r^{2}$.

In the new coordinates, $g_{00}$ component of the Schwarzschild solution takes the following form (the tilde is omitted),

$g_{00}=1-\frac{r_{g}}{r}+\frac{r_{g}^{2}}{2 r^{2}}+O\left(\frac{r_{g}^{3}}{r^{3}}\right)$

Taking the square root of $g_{00}$, we see that the classical gravitational potential turns out to be equal to

$$
\Phi^{\mathrm{c}}(r)=-\frac{G M}{r}+\frac{G^{2} M^{2}}{2 c^{2} r^{2}} .
$$

The post-Newtonian correction here is twice as small as that obtained in [1].

Thus, we arrive at the puzzling conclusion that in the classical limit, the quantum theory of gravity, being based on the Bohr correspondence principle, does not reproduce the Einstein theory it originates from.

However, before making such a conclusion, one should question relevance of the definition of gravitational potential through the scattering amplitudes. It may well turn out that the above discrepancy arises because of the incorrect choice of the quantum-field quantity to be traced back to the classical potential.

In what follows, I give an interpretation of the correspondence principle as applied to gravity, which naturally resolves the above paradox [2].

Let us note first of all that, from the formal point of view, the correspondence between any quantum theory and its classical original is most naturally established in terms of the effective action rather than the S-matrix. This is because the effective action (generating functional of the one-particle-irreducible Green functions) just coincides with the initial classical action in the tree approximation. In particular, the nonlinearity of classical theory (resulting, e.g., in $r_{g} / r$ power corrections to the Newton law in the General Relativity) is correctly reproduced by the trees. In the case of quantum gravity, there are still additional contributions of the order $\hbar^{0}$, coming from loop corrections. These are given by the gravitational form factors of the particles, which serve as building blocks for the gravitational potential. Instead of constructing the potential, however, let us consider them in the framework of the effective action method. From the point of view of this method, the $\hbar^{0}$-parts of the particle form factors, together with the proper quantum parts of order $\hbar$, describe the radiative corrections to the classical equations of motion of the gravitational field. It is non-vanishing of these terms that violates the usual Bohr correspondence. There is, however, one essential difference between the $\hbar^{0}$ loop terms and the nonlinear tree corrections. Consider, for instance, the first post-Newtonian cor- 


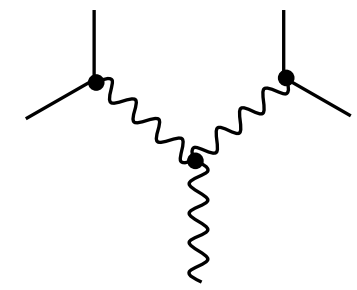

(a)
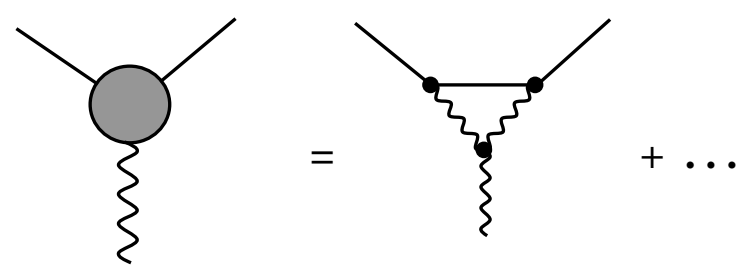

(b)

Figure 1. Diagrams contributing to the first postNewtonian correction. (a) The tree diagram occurring because of the nonlinearity of the Einstein equations. (b) The one-loop form factor. Wavy lines represent gravitons, solid lines scalar particles.

rection of the form

$$
\text { const } \frac{G^{2} M^{2}}{c^{2} r^{2}} .
$$

The "const" receives contributions from the tree diagram pictured in Fig. 11(a), as well as from the one-loop form factor, Fig. 1 (b). If the gravitational field is produced by only one particle of mass $M$, then the two contributions are of the same order of magnitude.

They are not, however, if the field is produced by a macroscopic body consisting of a large number $N$ of particles with mass $m=M / N$. Being responsible for the nonlinearity of Einstein equations, the tree diagram 1(a) is bilinear in the energy-momentum tensor $T^{\mu \nu}$ of the particles, while the loop diagram 1(b) is only linear (to be precise, it has only two particle operators attached). Therefore, when evaluated be- tween the $N$-particle states, the former is proportional to $(m \cdot N) \cdot(m \cdot N)=M^{2}$, while the latter, to $m^{2} \cdot N=M^{2} / N$. If, for instance, the solar gravitational field is considered, the quantum correction is suppressed by a factor of the order $m_{\text {proton }} / M_{\odot} \approx 10^{-57}$.

This fact suggests the following interpretation of the correspondence principle when applied to the case of gravity: the effective gravitational field produced by a macroscopic body of mass $M$ consisting of $N$ particles turns into the corresponding classical solution of the Einstein equations in the limit $M \rightarrow \infty, N \rightarrow \infty$.

It is clear from the above discussion that the use of the effective action is essential for this interpretation: the quantum potential defined with the help of the scattering amplitudes, being of the form

$$
\begin{aligned}
& \Phi^{\mathrm{q}}(r)=-N \frac{G(M / N)}{r}+c_{1} N \frac{G^{2}(M / N)^{2}}{c^{2} r^{2}} \\
& +c_{2} N \frac{G^{3}(M / N)^{3}}{c^{4} r^{3}}+\cdots,
\end{aligned}
$$

for a macroscopic ( $N$-particle) body of mass $M$, would fail to reproduce whatever classical potential other than Newtonian, since

$$
\Phi^{\mathrm{q}}(r) \rightarrow-\frac{G M}{r} \text { when } N \rightarrow \infty .
$$

Thus, the loop corrections of the order $\hbar^{0}$ are now considered on an equal footing with the tree corrections, and thereby are endowed with direct physical meaning as describing deviations of the spacetime metric from classical solutions of the Einstein equations in the case of finite $N$.

Like any other argument trying to assign physical meaning to the effective action, the above interpretation immediately runs up against the problem of its gauge dependence. In spite of being independent of the Planck constant, the $\hbar^{0}$ terms originating from the loop diagrams are not gauge-independent a priori. However, there is a strong evidence for that they are gaugeindependent nevertheless. Namely, as is demonstrated in full detail in [2], the $\hbar^{0}$ terms of the one-loop gravitational form factors of the scalar particle, contributing to the first post-Newtonian 
correction to the metric, turn out to be independent of the Feynman gauge parameter $\xi$ weighting the gauge condition. A very specific feature of this gauge dependence cancellation must be emphasized: it holds only for the $\hbar^{0}$ part of the form factors. For instance, the logarithmical part of the form factors (which is of the order $\hbar$ ) is gauge-dependent [ 3].

Actual value of the one-loop $\hbar^{0}$ contribution to the effective metric can be found with the help of the explicit expressions for the gravitational form factors of the scalar particle, obtained in Ref. [1]. In the momentum space,

$$
\begin{aligned}
& \delta g_{\mu \nu}(p)=-\frac{\pi^{2} G^{2} m^{2}}{c^{2} \sqrt{-p^{2}}} \\
& \times\left(3 \eta_{\mu \nu}+\frac{q_{\mu} q_{\nu}}{m^{2}}+\frac{7 p_{\mu} p_{\nu}}{p^{2}}\right),
\end{aligned}
$$

where $q_{\mu}$ is the 4-momentum of the scalar particle with mass $m$.

With the help of Eq. (11), one can find the effective gravitational potential of a spherically symmetric body with mass $M$, consisting of $N$ identical (scalar) particles with mass $m=M / N$, in the first post-Newtonian approximation. Namely, if the gravitational interaction of the constituent particles is not too strong (viz., if it can be represented as a series in powers of $G$ ), then one has, in the coordinate space

$$
\Phi^{\mathrm{eff}}(r)=-\frac{G M}{r}+\frac{G^{2} M^{2}}{2 c^{2} r^{2}}-\frac{G^{2} M^{2}}{N c^{2} r^{2}} .
$$

Indeed, the tree contribution to the effective field, represented in this equation by the first two terms, always coincides with the corresponding classical (Schwarzschild) solution. It is given, therefore, by Eq. (3), all the effects of the particle interactions are taken into account by identifying $M$ as the gravitational mass, while account of the interaction in the loop correction (the third term in Eq. (5)), which is itself of the order $G^{2}$, would give rise to terms of the order $G^{3}$, irrelevant to the present concern. Appearance of the factor $1 / N$ in the third term was explained earlier.

It was mentioned above that the loop contributions to the post-Newtonian corrections are normally highly suppressed: their relative value for the stars is of the order $10^{-56}-10^{-58}$. This differs, however, in the case of objects consisting of strongly interacting particle. In particular, in the limit of an infinitely strong interaction, the object is to be considered as a particle, i.e., one has to set $N=1$ in Eq. (5). The sign of the first post-Newtonian correction is then opposite to that given by the classical General Relativity.

An example of objects of this type is probably supplied by the black holes. Applicability of the above results to these objects depends on whether they can be considered as elementary particles. It seems that at least certain types of the black holes do behave like normal elementary particles [ 柾. It should be noted, however, that the very existence of the horizon is now under question. The potential $\Phi^{\mathrm{eff}}(r)$ may well turn out to be a regular function of $r$ when all the $\hbar^{0}$ loop corrections are taken into account.

If the notion of elementary particle is indeed applicable to the black holes, then the above results imply, in particular, that the emission of the gravitational waves by the black hole binaries is strongly affected by the quantum contributions already in the first post-Newtonian approximation. The LIGO and VIRGO gravitational wave detectors [5], which are now under construction, will hopefully bring light into this issue.

\section{REFERENCES}

1. J. F. Donoghue, Phys. Rev. Lett. 72 (1994) 2996; Phys. Rev. D50 (1994) 3874; Perturbative Dynamics of Quantum General Relativity, invited plenary talk at the "Eighth Marcel Grossmann Conference on General Relativity", Jerusalem (1997).

2. K. A. Kazakov, Class. Quantum Grav. 18 (2001) 1039.

3. K. A. Kazakov, Phys. Rev. D63 (2001) 044004.

4. C. F. E. Holzhey, F. Wilczek, Nucl. Phys. B380 (1992) 447.

5. Description of the LIGO and VIRGO projects as well as information about their current development is available at http://www.ligo.caltech.edu http://www.virgo.infn.it. 